\title{
Neuromyelitis Optica Spectrum Disorder Associated with Cryptogenic Organizing Pneumonia in a Young Patient
}

\author{
Irfan Shafiq, Ali Saeed Wahla, Mateen Haider Uzbeck, Naureen Khan, Zaid Zoumot \\ Cleveland Clinic, Abu Dhabi, UAE
}

Doi: 10.12890/2022_003105 - European Journal of Case Reports in Internal Medicine - ๑ EFIM 2022

Received: 05/12/2021

Accepted: 19/01/2022

Published: 08/02/2022

\begin{abstract}
How to cite this article: Shafiq I, Wahla AS, Uzbeck MH, Khan N, Zoumot Z. Neuromyelitis optica spectrum disorder associated with cryptogenic organizing
\end{abstract} pneumonia in a young patient. EJCRIM 2022;9: doi:10.12890/2022_003105.

Conflicts of Interests: The authors declare there are no competing interests.

This article is licensed under a Commons Attribution Non-Commercial 4.0 License

\section{ABSTRACT}

Neuromyelitis optica spectrum disorder (NMOSD) is associated with other autoimmune disorders and probably with cryptogenic organizing pneumonia (COP) as well. Here we present the case of a 14-year-old girl presenting with typical NMOSD together with radiological evidence of COP. Our case is unique as the previous two reports of this association were in elderly patients.

\section{LEARNING POINTS}

- There probably is an association between neuromyelitis optica spectrum disorder (NMOSD) and cryptogenic organizing pneumonia (COP).

- In contrast to previously published reports describing NMOSD and COP in two elderly patients, our case report highlights the fact that this disease combination can be present in young patients as well.

\section{KEYWORDS}

Neuromyelitis optica spectrum disorder, cryptogenic organizing pneumonia, young patient

\section{INTRODUCTION}

Neuromyelitis optica spectrum disorder (NMOSD) is a rare condition characterized by autoimmune-mediated inflammation and subsequent demyelination of the central nervous system ${ }^{[1]}$. The demyelination and axonal damage primarily target the optic nerves and spinal cord, but can also affect the brain and brainstem ${ }^{[2]}$. The incidence of NMOSD in females is 7.6 times higher than in males and onset is typically between 32 and 41 years of age ${ }^{[3]}$. NMOSD can be distinguished from multiple sclerosis and other central nervous system inflammatory disorders by the presence of the disease-specific aquaporin-4 (AQP4) antibody, which plays a direct role in the pathogenesis of NMOSD ${ }^{[4]}$. It is thought to be an idiopathic condition, but the literature has reported an association with autoimmune diseases such as Sjögren's syndrome sarcoidosis, antiphospholipid syndrome and systemic lupus erythematosus ${ }^{[5]}$.

Cryptogenic organizing pneumonia (COP), previously known as bronchiolitis obliterans organizing pneumonia (BOOP), is one of the idiopathic interstitial pneumonias that primarily affect the distal bronchioles, respiratory bronchioles, alveolar ducts and alveolar walls ${ }^{[6]}$. Men and women are affected equally, with the typical age of onset being the 50's and 60's ${ }^{[7]}$.

In this case report we describe an unusual presentation of NMOSD together with COP in a 14-year-old patient. Although a clear association between these two pathologies has not been reported previously, it is hypothesized that there is a potential link between them. Understanding this association is crucial for both diagnosis and management of patients who present with these rare conditions. 


\section{CASE DESCRIPTION}

We present the case of a 14-year-old girl who was referred to the neurology clinic with progressive decrease in the visual acuity of her right eye accompanied by pain on eye movement and frontal headache for the previous 2 months. The patient did not report any history of trauma, paresis, bladder or bowel dysfunction, or seizures. Her past medical and family history were non-contributory. She was otherwise healthy and up-to-date with her vaccinations. Physical examination showed right-sided afferent pupillary defect and pain on extraocular movement. Fundoscopic examination showed no papilledema. Based on clinical suspicion of optic neuritis, the patient was admitted and empirically started on pulsed methylprednisolone. Her brain MRI revealed inflammatory changes in the right optic nerve suggestive of right optic neuritis. MRI of the spine showed an incidental right heterogenous paravertebral mass from T12-L1 to L4-L5 with vertebral body scalloping. This was later proven to be a ganglioneuroma on CT-guided core needle biopsy. The CSF analysis was within normal limits.

Parts of the chest visualized on MRI also showed bilateral pulmonary basal non-homogeneous consolidation. These findings were further confirmed by a chest CT scan that showed peripheral ground-glass opacities involving the bilateral lower lobes; some had a denser outer rim, consistent with the reverse halo sign and hence were highly suggestive of an organizing pneumonia (Fig. 1).
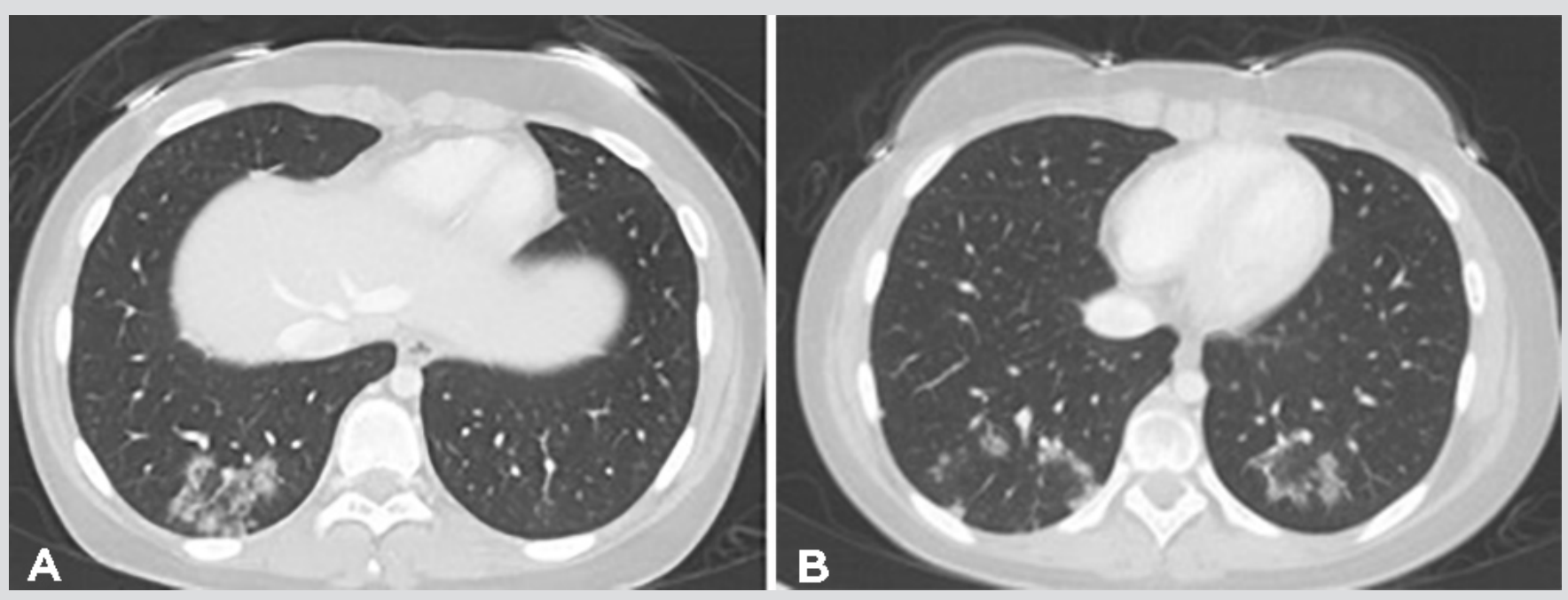

Figure 1. Ground-glass opacities in the periphery of the lower lobes prior to the start of immunosuppressive treatment, some of which demonstrate the reverse halo sign

Although a lung biopsy was considered for confirmation of the diagnosis of COP, given the patient's young age, lack of respiratory symptoms and typical radiological findings, the decision was made against it.

Blood work revealed positive aquaporin-4 antibodies (AQP4-IgG), negative anti-myelin oligodendrocyte glycoprotein (MOG), ANA, P-ANCA and C-ANCA. The MRI findings and the presence of AQP4-IgG were both consistent with a diagnosis of NMOSD.

The patient was subsequently treated with 5 days of IV methylprednisolone and five cycles of plasma exchange (Plex). Unfortunately, her vision did not improve but there was significant improvement in the radiological appearance of the lung lesions with complete resolution of previously seen opacities but the appearance of new nodules in a different location (Fig. 2).

Since the patient's vision did not improve, she was treated with rituximab which also had a very modest effect on her neurological symptoms. Two months after the rituximab treatment, CT of the chest again showed resolution of previously seen nodules with the appearance of new ones in a different location and with the characteristic appearance of organizing pneumonia (Fig. 3) including patchy airspace ground-glass and nodular opacities with subpleural and lower zone predominance and some with the reverse halo sign. It is of note that despite nonremitting neurological disease and the persistence of organizing pneumonia findings on CT scans, the patient had no respiratory symptoms at any point and had completely normal lung function.

\section{DISCUSSION}

This case is unusual and interesting as this young girl presented with symptoms of early onset NMOSD along with COP. Since the pathogenesis of NMSOD involves the development of antibodies against AQ4, which is also expressed in lung tissue, it is likely that this occurrence is a true association rather than a coincidence. Also, two previous case reports have shown this association, albeit in elderly patients [8, 9].

AQ4 is known to be present not only in airway epithelium but also in alveoli and its expression seems to be altered in several disease 
conditions, including asthma, chronic obstructive pulmonary disease and acute lung injury [10]; however, the exact role of AQ4 in the pathogenesis of these conditions, as well as COP, remains unclear. Although both NMSOD and COP can present as paraneoplastic conditions [9], no malignancy was found in our patient.

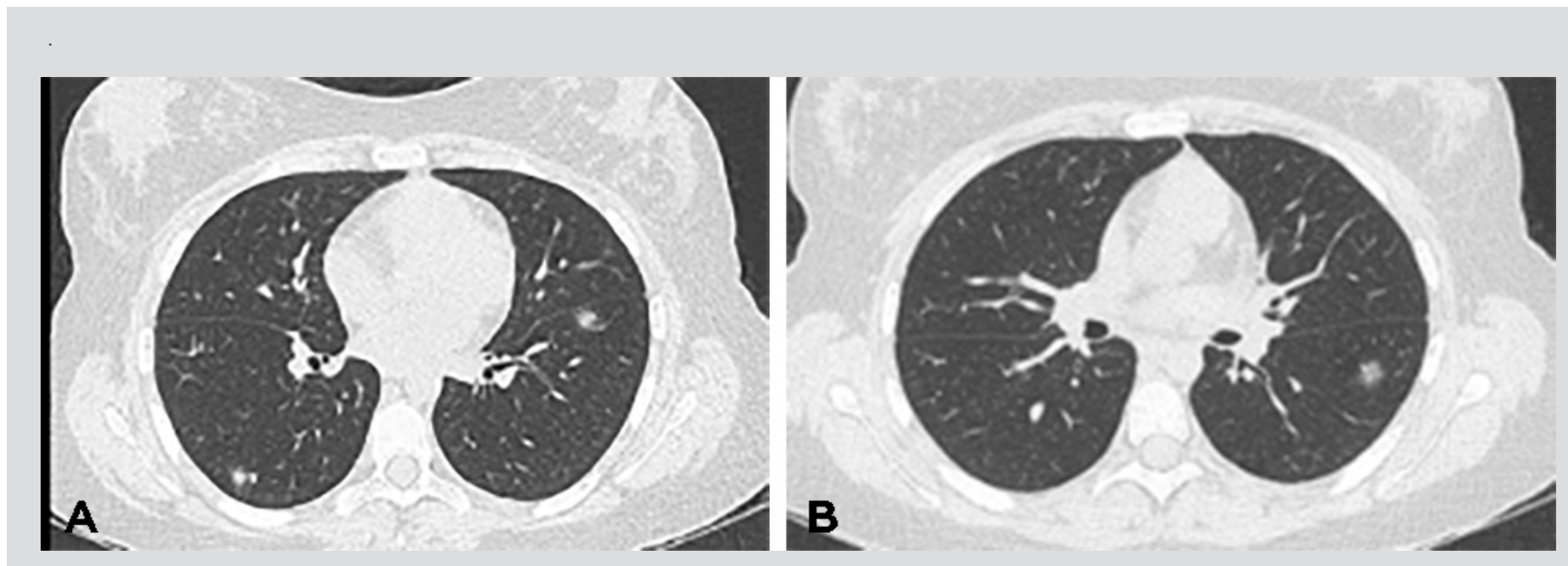

Figure 2. CT of the chest 4 weeks after systemic corticosteroids and plasma exchange showed resolution of previous opacities but the development of new nodules in different locations
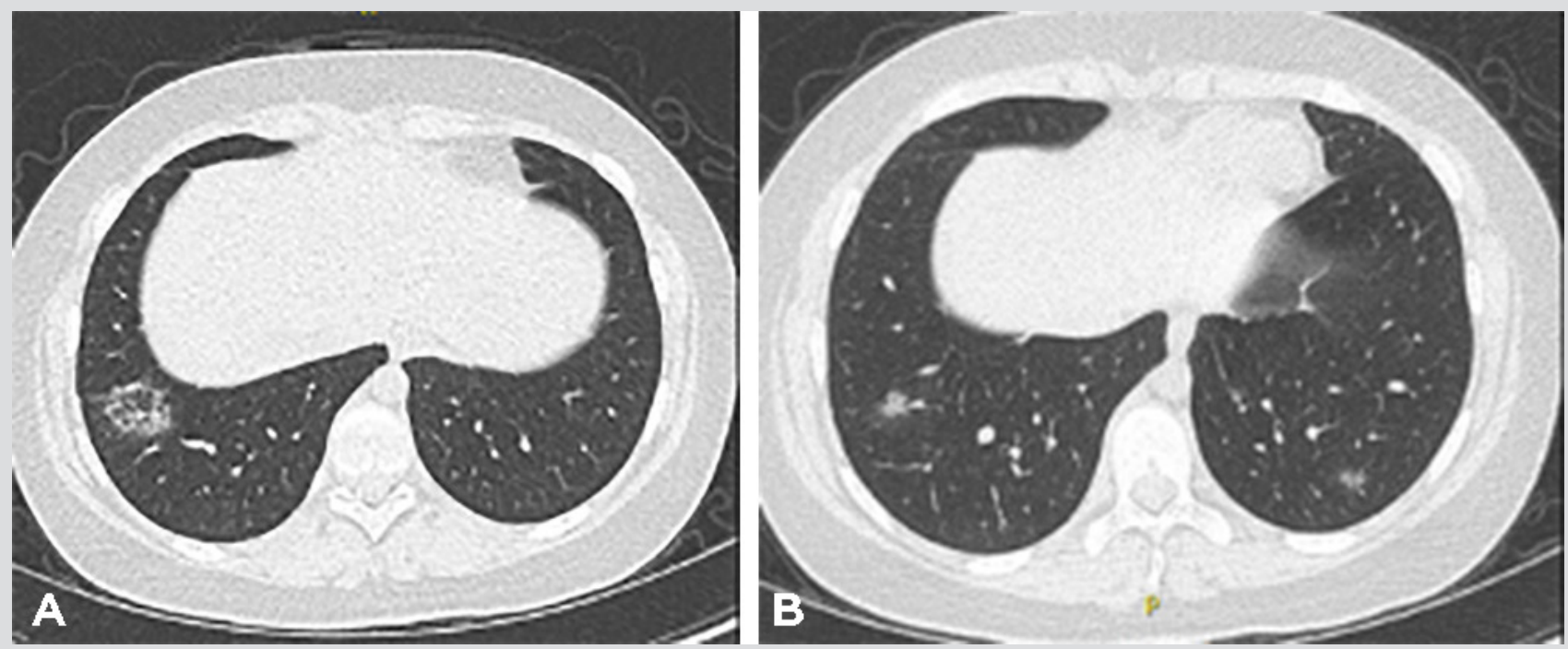

Figure 3. Further CT imaging after treatment with rituximab again showed resolution of previous opacities but the appearance of new nodular ground-glass opacities in the periphery of the lower lobes

The age at presentation of NMOSD directly correlates with its prognosis. Previous literature has shown that paediatric patients with earlyonset NMOSD have more debilitating symptoms, a higher number of relapses, and increased morbidity when compared with adult subjects. Although there are limited data on the mortality rate of patients with NMOSD, one study showed a high fatality rate of $15 \%$ in patients with early-onset disease ${ }^{[11]}$.

Similar to our case, one of the most commonly reported symptoms of NMOSD is acute onset of optic neuritis that can progress to visual loss. Other signs of NMOSD include transverse myelitis that can present with muscular weakness and bladder dysfunction, intractable hiccups, sleep disorders, neuroendocrine disorders, and seizures in children ${ }^{[12]}$. The assessment of clinically suspected NMOSD includes CNS neuroimaging, detection of AQP4 antibody, and cerebrospinal fluid analysis ${ }^{[4]}$.

NMOSD is initially managed with high-dose intravenous methylprednisolone. For patients who do not respond to glucocorticoids, treatment with plasma exchange is often effective. Since NMOSD has a relapsing course in $90 \%$ of patients and most deteriorate rapidly, early long- 
term immunotherapy with eculizumab, inebilizumab, rituximab or satralizumab is recommended to decrease the chance of relapse ${ }^{[13]}$. In a previous study conducted by Fragoso et al., it was observed that 43\% patients did not improve with azathioprine and daily prednisone. However, increasing the dose or switching the treatment regimen to rituximab, and plasma exchange resulted in optimal disease control in most patients ${ }^{[9]}$.

Our patient also presented with concomitant COP, a chronic inflammatory lung disease characterized by multiple ground-glass opacities on chest imaging and interstitial infiltration by mononuclear cells and macrophages on histology ${ }^{[14]}$. Since the mainstay of treatment for patients with COP is systemic glucocorticoid therapy and immunosuppression, we continued the patient's rituximab and corticosteroids ${ }^{[15]}$. There are limited data on any association between these two distinct conditions. However, AQP4 expression has been discovered in lung tissue, which might explain the link between COP and NMOSD. Furthermore, similar to the processes of autoimmune reaction in patients with underlying malignancy and paraneoplastic disorders, an immune response to pulmonary antigens might stimulate an immunological reaction against cross-reactive nervous system antigens on the myelin sheath. The clinical, biochemical and MRI characteristics of NMOSD change according to the age of onset, indicating that variations in aetiology and therapy should be explored further.

\section{REFERENCES}

1. Paul S, Mondal GP, Bhattacharyya R, Ghosh KC, Bhat IA. Neuromyelitis optica spectrum disorders. J Neurol Sci 2021;420(2):169-176

2. Wingerchuk DM, Lennon VA, Lucchinetti CF, Pittock SJ, Weinshenker BG. The spectrum of neuromyelitis optica. Lancet Neurol 2007;6(9):805-815.

3. Papp V, Magyari M, Aktas O, Berger T, Broadley SA, Cabre P, et al. Worldwide incidence and prevalence of neuromyelitis optica: a systematic review. Neurology 2021;96:59-77. Tan CT. International consensus diagnostic criteria for neuromyelitis optica spectrum disorders. Neurology 2016;86(5):491-492.

5. Iyer A, Elsone L, Appleton R, Jacob A. A review of the current literature and a guide to the early diagnosis of autoimmune disorders associated with neuromyelitis optica. Autoimmunity 2014;47(3):154-161.

6. Cordier JF. Cryptogenic organising pneumonia. Eur Respir J 2006;28(2):422-446.

7. Gudmundsson G, Sveinsson O, Isaksson HJ, Jonsson S, Frodadottir H, Aspelund T. Epidemiology of organising pneumonia in Iceland. Thorax 2006;61(9):805-808.

8. Ruisanchez Nieva A, Sanchez Menoyo JL, Escalza Cortina I, Foncea Beti N, Gomez Beldarrain M, Garcia-Monco JC. Neuromyelitis optica spectrum disorder in the elderly associated with bronchiolitis obliterans organizing pneumonia. Neurol Clin Pract 2017 Oct;7(5):404-406.

9. Oliveira R, Oliveira S. Letter to the Editor - Late-onset neuromyelitis optica associated with cryptogenic organizing pneumonia. Mult Scler Relat Disord 2019 Nov;36:101398.

10. Wittekindt OH, Dietl P. Aquaporins in the lung. Pflüg Arch 2019 Apr 1:471(4):519-532.

11. Fragoso YD, Sousa NAC, Saad T, Alves-Leon SV, Pimentel MLV, Goncalves MVM, et al. Clinical characteristics of patients with neuromyelitis optica spectrum disorders with early onset. J Child Neurol 2019;34(9):487-490.

12. Ghezzi A, Bergamaschi R, Martinelli V, Trojano M, Tola MR, Merelli E, et al. Clinical characteristics, course and prognosis of relapsing Devic's neuromyelitis optica. J Neurol 2004;251(1):47-52.

13. Sellner J, Boggild M, Clanet M, Hintzen RQ, Illes Z, Montalban X, et al. EFNS guidelines on diagnosis and management of neuromyelitis optica. Eur J Neurol 2010;17(8):10191032.

14. Cordier JF, Loire R, Brune J. Idiopathic bronchiolitis obliterans organizing pneumonia. Definition of characteristic clinical profiles in a series of 16 patients. Chest 1989;96(5):999-1004.

15. Wells AU, Hirani N. Interstitial lung disease guideline: The British Thoracic Society in collaboration with the Thoracic Society of Australia and New Zealand and the Irish Thoracic Society. Thorax 2008;63(suppl. 5):v1-58. 